Rev. Int. Contam. Ambie. 38, 1-10, 2022

https://doi.org/10.20937/RICA.53995

\title{
RETENCIÓN DE CONTAMINANTES AROMÁTICOS LÍQUIDOS MEDIANTE HIDROTALCITAS, ÓXIDOS MIXTOS DERIVADOS E HIDROTALCITAS RECONSTRUIDAS
}

Retention of liquid aromatic contaminants by hydrotalcites, derived mixed oxides, and reconstructed hydrotalcites Franchescoli D. VELÁZQUEZ-HERRERA ${ }^{1}$, Álvaro SAMPIERI $^{2}$,
Patricia PAREDES-CARRERA ${ }^{3}$ y Geolar FETTER ${ }^{1 *}$

${ }^{1}$ Facultad de Ciencias Químicas, Benemérita Universidad Autónoma de Puebla, Ciudad Universitaria, 72570 Puebla, Puebla, México.

${ }^{2}$ Facultad de Ingeniería Química, Benemérita Universidad Autónoma de Puebla, Ciudad Universitaria, 72570 Puebla, Puebla, México.

${ }^{3}$ Escuela Superior de Ingeniería Química e Industrias Extractivas, Instituto Politécnico Nacional, Zacatenco, 07738 Ciudad de México, México.

*Autor para correspondencia: geolarfetter@yahoo.com.mx

(Recibido: abril de 2020; aceptado: enero de 2021)

Palabras clave: hidróxidos dobles laminares, adsorbentes, contaminantes orgánicos, compuestos aromáticos, BTEX-fenol

\section{RESUMEN}

Las arcillas aniónicas o hidrotalcitas son materiales multifuncionales que permiten optimizar procesos como la adsorción de contaminantes, entre los cuales los compuestos orgánicos aromáticos representan un grave problema ambiental, ya que se encuentran dispersos en aguas residuales generadas normalmente por la industria petroquímica. En este trabajo se presenta la síntesis de hidrotalcitas y de óxidos mixtos derivados de éstas, empleados en la adsorción de contaminantes orgánicos de tipo BTEX-fenol presentes en medio acuoso. Los resultados se compararon con los de las hidrotalcitas reconstruidas en presencia de BTEX-fenol. Se encontró que las propiedades adsorptivas dependen fundamentalmente de la composición química de los materiales y de la estructura. Si bien las hidrotalcitas iniciales y los óxidos mixtos resultaron ser adsorbentes eficientes, las hidrotalcitas reestructuradas fueron mucho más activas.

Key words: layered double hydroxides, adsorbents, organic pollutants, aromatic compounds, BTEX-phenol

\begin{abstract}
Anionic clays or hydrotalcites are multifunctional materials that can improve processes such as adsorption of contaminants. Among these, aromatic organic compounds represent an environmental problem, since they are dispersed in wastewater usually generated by the petrochemical industry. This work presents the synthesis of hydrotalcites and mixed oxides derived from hydrotalcites, used in the adsorption of organic pollutants of the BTEX-phenol present in aqueous media. The results were compared with those of the reconstructed hydrotalcites in presence of BTEX-phenol.
\end{abstract}


It was found that the adsorptive properties depend on the chemical composition and the structural properties of the materials. Although the initial hydrotalcites and the derived mixed oxides were efficient adsorbents, the regenerated hydrotalcites were much more active.

\section{INTRODUCCIÓN}

Los compuestos orgánicos volátiles (COV) son aquellos hidrocarburos que se encuentran en estado gaseoso o que son muy volátiles en condiciones estándar de temperatura y presión. Suelen presentar una cadena con número de carbonos inferior a 12 , y contienen otros elementos como oxígeno, flúor, cloro, bromo, azufre o nitrógeno. Son los principales contaminantes del ambiente ( $\mathrm{Li}$ et al. 2020) y son reconocidos por contribuir a la destrucción de la capa de ozono afectando la salud humana (Blanch-Raga et al. 2014).

Los problemas ambientales asociados a los $\mathrm{COV}$ han sido la fuerza impulsora para la investigación sostenible, fundamental y aplicada en el área de remediación ambiental de contaminantes como benceno, tolueno, etilbenceno, xilenos (BTEX) y fenol. Esta mezcla de contaminantes proviene, normalmente, de desechos de la industria petrolera y están presentes en aguas de desecho (Makhathini y Rathilal 2017). Por lo tanto, un método eficiente de tratamiento debe considerar el tratamiento de todos los compuestos a la vez.

Los métodos utilizados convencionalmente para tratar estos contaminantes contemplan diversos procesos físicos y químicos, de los cuales la adsorción es el más empleado debido a que presenta una alta selectividad para determinados compuestos y es útil cuando estos se encuentran en fase acuosa. Además, el método de adsorción resulta más económico que los demás procesos. Los materiales más empleados en este tipo de proceso son las zeolitas, los carbones activados y también algunos polímeros; sin embargo, en la actualidad se ha planteado el uso de nuevos materiales que permitan mejorar la adsorción de $\mathrm{COV}$ (Dudek et al. 2012), siendo uno de estos materiales las hidrotalcitas (Sampieri et al. 2018, Jurado-Dávila et al. 2020).

Las hidrotalcitas son arcillas naturales o sintéticas formadas por láminas apiladas cargadas positivamente, cuya carga es compensada por aniones que se localizan en la región interlaminar, en la cual también se depositan moléculas de agua que le confieren mayor estabilidad a la estructura. Su fórmula química general es:
$\left[\mathrm{M}^{2+}{ }_{1-\mathrm{x}} \mathrm{M}^{3+}{ }_{\mathrm{x}}(\mathrm{OH})_{2}\right]\left(\mathrm{X}^{\mathrm{m}-}\right)_{\mathrm{x} / \mathrm{m} \cdot \mathrm{nH}} \cdot \mathrm{H}_{2} \mathrm{O}$

donde $\mathrm{M}^{2+} \mathrm{y} \mathrm{M}^{3+}$ son metales divalentes y trivalentes, respectivamente; $\mathrm{X}^{\mathrm{m}-}$ es un anión de compensación con carga $\mathrm{m}-$; $\mathrm{x}$ representa la relación metálica $\mathrm{M}^{3+}$ / $\left(\mathrm{M}^{3+}+\mathrm{M}^{2+}\right)$, y n es el número de moléculas de agua (Zarazúa-Aguilar et al. 2018).

Estos materiales pueden albergar en su estructura diversos tipos de cationes metálicos, siendo los más comunes magnesio y aluminio. El zinc y el cobre están siendo estudiados actualmente en la formación de hidrotalcitas para aplicaciones médicas (Lobo-Sánchez et al. 2018, León-Vallejo et al. 2019, Segura-Pérez et al. 2020). Existe una gran variedad de aniones que se pueden integrar a la estructura de las hidrotalcitas, desde especies simples como los halogenuros hasta moléculas complejas como el ADN (Mishra et al. 2018).

En el empleo como catalizadores básicos, las hidrotalcitas de $\mathrm{Mg}$ y $\mathrm{Al}$ han resultado muy efectivas en reacciones de condensación aldólica de la acetona (Sommer et al. 2006) o en la producción de compuestos anticancerígenos como los cromenos (VelázquezHerrera et al. 2020). Con relación a su utilización como adsorbentes, las hidrotalcitas más eficaces se han modificado utilizando procesos difíciles y costosos. En este contexto, las hidrotalcitas fluoradas se utilizan para retención de trihalometanos (Morales et al. 2018), las soportadas en sílice mesoporosa para la retención del cromo (VI) presente en agua (Pérez et al. 2015) y las soportadas en hidroxiapatita para la remoción de fluoruros (Sani et al. 2016).

A pesar de que varios autores reportan hidrotalcitas obtenidas por métodos menos costosos para la retención de compuestos orgánicos, son pocos los estudios que reportan hidrotalcitas para la remoción de VOC en sistemas acuosos (Chaillot et al. 2020) e inexitentes los referentes a la adsorción de mezclas multicomponentes como BTEX y fenol. Por lo tanto, en este trabajo, se sintetizaron hidrotalcitas di y trimetálicas de $\mathrm{Mg}, \mathrm{Zn}$ y Al, y se probaron como adsorbentes para remover compuestos orgánicos en medio acuoso (de una mezcla conformada por benceno, tolueno, etilbenceno, xilenos y fenol), y se estudió el efecto de la composición de los materiales en la capacidad de retención de dichos compuestos 
orgánicos. Los resultados se compararon con muestras de óxidos mixtos derivados de la calcinación de hidrotalcitas y con hidrotalcitas reconstruidas.

\section{MATERIALES Y MÉTODOS}

\section{Síntesis de las hidrotalcitas}

Se sintetizaron hidrotalcitas de $\mathrm{Mg} / \mathrm{Al}, \mathrm{Zn} / \mathrm{Al}$ y $\mathrm{Zn}$ $\mathrm{Mg} / \mathrm{Al}$ con una relación molar $\mathrm{M}^{2+}: \mathrm{M}^{3+}$ de $2: 1$ a partir de una solución $1.5 \mathrm{M}$ de las sales de los nitratos de $\mathrm{Al}$ (Sigma-Aldrich, $98 \%$ ) y de Mg (Sigma-Aldrich, $98 \%$ ) y/o Zn (Sigma-Aldrich, 98 \%), y de otra solución $1.5 \mathrm{M}$ de hidróxido de amonio (Baker, 29\%) como agente precipitante. Se escogieron las sales de nitrato para que los aniones de compensación de cargas fueran nitratos, los cuales podrían facilitar el intercambio con los BTEX-fenol. Cada solución se colocó en una bureta y luego se agregaron lenta y simultáneamente en un vaso de precipitados que contenía $10 \mathrm{~mL}$ de agua destilada. El proceso de coprecipitación se mantuvo en agitación constante a temperatura ambiente y se controló el flujo de las soluciones de cada una de las buretas, a fin de que el $\mathrm{pH}$ se mantuviera constante e igual a 8. Una vez que se agotó la solución de las sales metálicas, la mezcla se trató por irradiación con microondas por 10 min a $200 \mathrm{~W}$ y $130 \mathrm{rpm}$ de agitación en un reactor modelo MIC-I de Sistemas y Equipos de Vidrio. Después de la etapa de cristalización por irradiación, los sólidos fueron separados de la solución y lavados con agua destilada (aproximadamente $800 \mathrm{~mL} / \mathrm{g}$ ) varias veces hasta que el $\mathrm{pH}$ del agua de lavados permaneció constante. Posteriormente se secaron los sólidos en una estufa a $60^{\circ} \mathrm{C}$ por $48 \mathrm{~h}$. Las muestras se nombraron según sus composiciones químicas como MA para la hidrotalcita de $\mathrm{Mg} / \mathrm{Al}$, ZA para la de $\mathrm{Zn} / \mathrm{Al}$ y ZMA para la trimetálica de $\mathrm{Zn}-\mathrm{Mg} / \mathrm{Al}$.

\section{Síntesis de los óxidos mixtos derivados de las hidrotalcitas}

Una parte de las hidrotalcitas obtenidas por el procedimiento descrito anteriormente fue calcinada por $6 \mathrm{~h}$ en un horno tubular (ESEVE HT 100) programado de tal forma que la temperatura aumentara $1{ }^{\circ} \mathrm{C} / \mathrm{min}$ hasta alcanzar $550{ }^{\circ} \mathrm{C}$ en presencia de flujo de aire. Los óxidos mixtos obtenidos por calcinación de las hidrotalcitas fueron nombrados según la nomenclatura de la hidrotalcita, adicionada la letra $\mathrm{C}$ : muestras MAC, ZAC y ZMAC.

\section{Obtención de las hidrotalcitas reconstruidas}

La reconstrucción de las hidrotalcitas se realizó por medio de mezclas de $20 \mathrm{~mL}$ de los óxidos mixtos con una solución que contenía $0.05 \mathrm{mg} / \mathrm{mL}$ de BTEXfenol $(0.009 \mathrm{mg} / \mathrm{mL}$ de benceno, $0.01 \mathrm{mg} / \mathrm{mL}$ de tolueno, $0.01 \mathrm{mg} / \mathrm{mL}$ de etilbenceno, $0.01 \mathrm{mg} / \mathrm{mL}$ de xilenos y $0.009 \mathrm{mg} / \mathrm{mL}$ de fenol). Los $\mathrm{pH}$ de las mezclas se ajustaron a 9 con una solución $1 \mathrm{M}$ de $\mathrm{NaOH}$. Las mezclas se sometieron a un tratamiento ultrasónico con un equipo Branson B5510DTH que operó a $40 \mathrm{kHz}$ por $10 \mathrm{~min}$. Los sólidos fueron recuperados por decantación y secados en estufa a $60{ }^{\circ} \mathrm{C}$, resultando en las muestras MAR, ZAR y ZMAR, donde $\mathrm{R}$ significa reconstruida.

\section{Caracterización de los sólidos}

Los patrones de difracción de rayos $\mathrm{X}$ de las muestras se obtuvieron en un difractómetro Bruker D8 Discover equipado con un ánodo de cobre $(\lambda=$ $1.54056 \AA$ ) y un espejo de tipo Göbel. Los ángulos de escaneo fueron de 5 a $70^{\circ} 2 \theta$ con un tamaño de paso de $0.025^{\circ} 2 \theta$ y un tiempo de incidencia de $36 \mathrm{~s}$. El tamaño de cristalito se determinó por la ecuación de Scherrer:

$L(h k 1)=K \lambda /(B(\theta) \cos \theta)$

donde $L$ es el tamaño promedio de cristalito referente a un determinado plano, $K$ es el factor de forma $(0.89), \lambda$ es la longitud promedio de la radiación de $\mathrm{CuK} \alpha(\lambda=1.54056 \AA), B(\theta)$ es la longitud a la altura media (FWHM, por sus siglas en inglés) del pico seleccionado y $\theta$ es el ángulo de difracción. Los espectros infrarrojos (FTIR) en la región de $4000-650 \mathrm{~cm}^{-1}$ se obtuvieron con un equipo Perkin-Elmer Spectrum One FTIR Spectrometer 74070 mediante reflectancia total atenuada (ATR). Las composiciones elementales de las hidrotalcitas se obtuvieron con un microscopio electrónico de barrido JEOL JSM-6610LV acoplado a un detector de dispersión de energía de rayos $\mathrm{X}$ (EDS). La determinación del área superficial específica y la distribución del tamaño de poro se realizó en un equipo Micromeritics ASAP 2460. Para dicho análisis, $0.2 \mathrm{~g}$ de material se desgasificaron a $80^{\circ} \mathrm{C}$ por $2 \mathrm{~h}$ y a $150^{\circ} \mathrm{C}$ por $12 \mathrm{~h}$ antes del análisis. El área superficial específica se calculó mediante el método BET y la distribución del diámetro de poro se determinó por el método BJH aplicado a la isoterma de desorción de nitrógeno.

\section{Adsorción de BTEX-fenol por las hidrotalcitas y óxidos mixtos}

Se colocaron $10 \mathrm{mg}$ de hidrotalcita o de los óxidos mixtos en frascos de $15 \mathrm{~mL}$ de capacidad, a los cuales se adicionaron $10 \mathrm{~mL}$ de una solución de $0.05 \mathrm{mg} / \mathrm{mL}$ de BTEX-fenol $(0.009 \mathrm{mg} / \mathrm{mL}$ de 
benceno, $0.01 \mathrm{mg} / \mathrm{mL}$ de tolueno, $0.01 \mathrm{mg} / \mathrm{mL}$ de etilbenceno, $0.01 \mathrm{mg} / \mathrm{mL}$ de xilenos y $0.009 \mathrm{mg} /$ $\mathrm{mL}$ de fenol). Los frascos se agitaron en un agitador orbital Sea Star Digital Orbital Shaker a 300 rpm. Durante el proceso, se recolectaron alícuotas en tiempos de $1,5,10,20,30,60,120,180,240,1440,2880$ y $7200 \mathrm{~min}$. Las alícuotas se analizaron en un espectrofotómetro GBC cintra 20 a $\lambda=270 \mathrm{~nm}$. A esta longitud de onda se identifican todos los compuestos orgánicos en estudio. La cuantificación de los compuestos retenidos en los materiales se efectuó por diferencia entre la cantidad inicial y el remanente en la solución empleando una curva de calibración.

\section{Adsorción de la mezcla BTEX-fenol por recons- trucción estructural}

Se colocaron $20 \mathrm{mg}$ de material calcinado (muestras MAC, ZAC y ZMAC) en frascos de $25 \mathrm{~mL}$ de capacidad. Después se adicionaron $20 \mathrm{~mL}$ de una solución con $0.05 \mathrm{mg} / \mathrm{mL}$ de BTEX-fenol, en las mismas proporciones y con el procedimiento descrito anteriormente. Se tomaron alícuotas durante el proceso. La primera alícuota se obtuvo de la solución estándar, correspondiendo al tiempo 0; la segunda alícuota (10 $\mathrm{min})$ se tomó al finalizar el tratamiento con ultrasonido, y la tercera alícuota se tomó a los $20 \mathrm{~min}$. Las alícuotas subsecuentes se tomaron al resto de los tiempos descritos. Los análisis cuantitativos de retención de contaminantes se efectuaron en la forma descrita anteriormente.

\section{RESULTADOS Y DISCUSIÓN}

Los difractogramas de rayos- $\mathrm{X}$ de las muestras (Fig. 1) revelan los picos correspondientes a los planos de difracción $(003,006,012,110$ y 113) característicos de los compuestos tipo hidrotalcita y acordes con el patrón de referencia JCPDS 00-0140191 (Sommer et al. 2010). En el difractograma de la muestra MA se observa, además, un pico de difracción a $18.3^{\circ} 2 \Theta$, característico de la gibbsita (JCPDS 01-076-1782) presente como impureza, teniendo en cuenta que el $\mathrm{Al}$ se precipita en forma de hidróxido (gibbsita) a valores de $\mathrm{pH}$ menores a 9 (Tamura et al. 2006).

En todas las muestras se puede observar la presencia de picos dobles correspondientes a los planos 003 y 006 , cuyo efecto es muy visible en la muestra ZA. Este fenómeno se atribuye a la segregación de aniones nitrato, que ocurre sobre todo con hidrotalcitas que contienen zinc (Velázquez-Herrera y Fetter 2020). Las distancias interlaminares $d_{003}$ y $d_{003} *$ corresponden a los dobletes del pico de difracción (003) en los ángulos 10 y $11^{\circ} 2 \theta$ con valores de aproximadamente 8.8 y $7.7 \AA$ (Cuadro I) $\mathrm{y}$ se atribuyen a la presencia de iones nitrato $\mathrm{y}$ carbonato, respectivamente (Velázquez-Herrera et al. 2018). Los carbonatos presentes son el resultado de la disolución de $\mathrm{CO}_{2}$ atmosférico durante el proceso de síntesis.

En el cuadro I se reportan los valores de los parámetros estructurales $a$ y $c$, donde $c$ se relaciona con las distancias interlaminares de los materiales y $a$ con las distancias inter-metálicas de los cationes de las láminas. Las hidrotalcitas ZA y MZA presentan parámetros $a=3.06 \AA \mathrm{y} c=26.5 \AA$, mientras que la muestra MA presenta parámetros estructurales ligeramente menores, siendo $a=3.03 \AA$ y $c=25.9 \AA$. La disminución en el parámetro $c$ puede estar asociada con una mayor presencia de iones carbonato en la muestra que contiene magnesio, mientras que la disminución en el parámetro $a$ probablemente se deba a que el $\mathrm{Mg}^{2+}(0.65 \AA)$ posee un radio iónico menor que el $\mathrm{Zn}^{2+}(0.74 \AA)$, lo cual reduce así, las distancias intermetálicas en la red. Hay que considerar también que una mayor cantidad de aluminio contribuye a la disminución del valor del parámetro $a$.

CUADRO I. PARÁMETROS ESTRUCTURALES $(a, c$, $\left.c^{*}\right)$, DISTANCIAS INTERLAMINARES DE LAS HIDROTALCITAS E HIDROTALCITAS RECONSTRUIDAS ( $\mathrm{d}_{003}, \mathrm{~d}_{003}$ ), Y TAMAÑOS DE DOMINIOS CRISTALINOS (L) DE LAS HIDROTALCITAS, ÓXIDOS MIXTOS E HIDROTALCITAS RECONSTRUIDAS.

\begin{tabular}{lcccccc}
\hline Muestra & $\begin{array}{c}d_{003} \\
(\AA)\end{array}$ & $\begin{array}{c}d_{003}^{*} \\
(\AA)\end{array}$ & $\begin{array}{c}a \\
(\AA)\end{array}$ & $\begin{array}{c}c \\
(\AA)\end{array}$ & $\begin{array}{c}c^{*} \\
(\AA)\end{array}$ & $\begin{array}{c}\text { L } \\
(\AA)\end{array}$ \\
\hline MA & 8.6 & 7.6 & 3.03 & 25.9 & 22.8 & 80 \\
ZA & 8.8 & 7.6 & 3.06 & 26.5 & 22.9 & 130 \\
ZMA & 8.8 & 7.7 & 3.06 & 26.5 & 23.0 & 180 \\
MAC & NA & NA & NA & NA & NA & 320 \\
ZAC & NA & NA & NA & NA & NA & 370 \\
ZMAC & NA & NA & NA & NA & NA & 490 \\
MAR & - & 7.6 & 3.06 & - & 22.8 & 123 \\
ZAR & - & 7.6 & 3.08 & - & 22.8 & 104 \\
ZMAR & - & - & - & - & - & - \\
\hline
\end{tabular}

*Valores obtenidos a partir del segundo pico de difracción o codo relativos al índice de Miller 003.

MA, ZA y ZMA: hidrotalcitas de Mg/Al, Zn/Al y trimetálica de $\mathrm{Zn}-\mathrm{Mg} / \mathrm{Al}$, respectivamente; MAC, ZAC y ZMAC: hidrotalcitas calcinadas de $\mathrm{Mg} / \mathrm{Al}, \mathrm{Zn} / \mathrm{Al}$ y trimetálica de $\mathrm{Zn}-\mathrm{Mg} / \mathrm{Al}$, respectivamente; MAR, ZAR y ZMAR: hidrotalcitas reconstruidas de $\mathrm{Mg} / \mathrm{Al}, \mathrm{Zn} / \mathrm{Al}$ y trimetálica de $\mathrm{Zn}-\mathrm{Mg} / \mathrm{Al}$, respectivamente; NA: no aplica. 
En el cuadro II se aprecia que la relación metálica molar para la muestra MA presenta un valor de 1.0, menor que los valores de las otras dos muestras, cercanos al valor nominal de 2 . Esta baja relación molar para la muestra MA sugiere, efectivamente, que una mayor cantidad de aluminio se integra a la red. No obstante, parte del aluminio puede estar asociada a la formación de gibbsita, presente en esta muestra como impureza.

CUADRO II. COMPOSICIÓN ELEMENTAL (\% EN MASA) OBTENIDA POR EDS Y RELACIÓN METÁLICA MOLAR $(\mathrm{Mg}+\mathrm{Zn} / \mathrm{Al})$ DE LAS HIDROTALCITAS.

\begin{tabular}{lccccc}
\hline Muestra & $\mathrm{O}$ & $\mathrm{Mg}$ & $\mathrm{Al}$ & $\mathrm{Zn}$ & $\begin{array}{c}\text { Relación molar } \\
\mathrm{Mg}+\mathrm{Zn} / \mathrm{Al}\end{array}$ \\
\hline MA & 85.24 & 6.61 & 8.15 & - & 1.0 \\
ZA & 60.29 & - & 5.39 & 34.31 & 2.5 \\
ZMA & 66.68 & 3.22 & 7.00 & 23.11 & 1.9 \\
\hline
\end{tabular}

EDS: detector de dispersión de energía de rayos X; MA, ZA y ZMA: hidrotalcitas de $\mathrm{Mg} / \mathrm{Al}, \mathrm{Zn} / \mathrm{Al}$ y trimetálica de $\mathrm{Zn}-\mathrm{Mg}$ / $\mathrm{Al}$, respectivamente.

Los difractogramas de los materiales calcinados (Fig. 1) revelan la transformación de las hidrotalcitas en los respectivos óxidos mixtos. En ellos se aprecian los principales picos característicos del óxido de zinc (zincita) a $31.8,34.5$ y $36.3^{\circ} 2 \theta$ de acuerdo con la ficha JCPDS 01-076-1782. Por la estrechez de

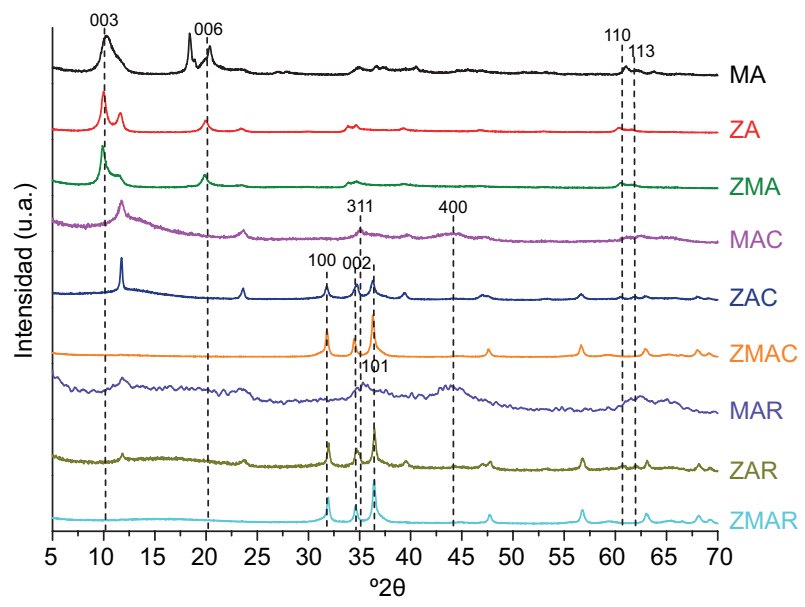

Fig. 1. Difractogramas de rayos $\mathrm{X}$ de las hidrotalcitas (muestras MA, ZA y ZMA), de los óxidos mixtos derivados de las hidrotalcitas (MAC, ZAC y ZMAC) y de las hidrotalcitas reconstruidas (MAR, ZAR y ZMAR). los picos de difracción se puede deducir el grado de cristalinidad de los óxidos resultantes, considerando que picos más angostos determinan mejor cristalinidad. Así, la muestra ZMAC es la que presenta mayor tamaño de cristales, seguida por la ZAC y la MAC. Por lo tanto, los tamaños de los cristales dependen de la composición química de las hidrotalcitas. En este contexto, el $\mathrm{Mg}$ en la hidrotalcita dimetálica favorece menores partículas, en tanto que el $\mathrm{Zn}$ favorece la formación de cristales de mayor tamaño, ya sea que provenga de la hidrotalcita dimetálica o trimetálica. Además, hay que considerar que el tamaño de cristal de las muestras iniciales de hidrotalcitas desempeña un papel importante en la formación de óxidos mixtos, como se reporta en el cuadro I, para los tamaños de dominios cristalinos de las muestras. Se observa que los valores decrecen desde $490 \AA$ para la muestra ZMAC hasta llegar al valor mínimo de $320 \AA$ para la MAC, pasando por $370 \AA \AA$ para la muestra ZAC, de acuerdo con los tamaños de los cristalitos de las hidrotalcitas, los cuales disminuyen en el mismo orden confirmando que la composición química determina, primordialmente, la cristalinidad de las muestras.

El difractograma del material reconstruido MAR (Fig. 1) revela la presencia de los picos poco intensos y anchos correspondientes a una hidrotalcita, indicando que se reconstruye de forma muy irregular debido a la presencia de contaminantes orgánicos que obstruyen el crecimiento de los cristales. El difractograma de la muestra reconstruida ZAR revela la presencia de picos característicos de la hidrotalcita y picos correspondientes al óxido de zinc; por lo tanto, en este caso, la reconstrucción de la hidrotalcita ocurre parcialmente, presentando características similares a la muestra MAR, en tanto que la reconstrucción de hidrotalcita en la muestra ZMAR se dificulta aún más, ya que sólo se aprecian los picos correspondientes a los óxidos. En este caso es probable que la hidrotalcita se componga de partículas mucho más pequeñas que las de las otras dos muestras, seguramente en escala nano. Estas consideraciones poden ser soportadas por los tamaños de dominios cristalinos (Cuadro I), cuyos valores siguen un orden decreciente de 123 a $104 \AA$ para las muestras MAR y ZAR, respectivamente, esto sin evaluar el tamaño de cristalito para la muestra ZMAR dada la baja intensidad de los picos de difracción.

A partir de la presencia de los picos de difracción también se puede deducir la estabilidad térmica de las muestras. Se observa que las hidrotalcitas ZA y MA no se descomponen totalmente, ya que en las muestras calcinadas ZAC y MAC aún se presentan los picos 003 y 006 característicos de hidrotalcitas, 
lo cual indica que la hidrotalcita de MgAl es más estable que la de $\mathrm{ZnAl}$ y que la trimetálica de $\mathrm{ZnMgAl}$ (Smoláková et al. 2017, León-Vallejo et al. 2019). Asimismo, se observa un desfasamiento de los picos 003 y 006 respecto a la hidrotalcitas no calcinadas, lo cual indica que los aniones de $\mathrm{NO}_{3}{ }^{2-}$ se descomponen a menor temperatura que los iones $\mathrm{CO}_{3}{ }^{2-}$. Por otro lado, la posición del pico 003 para las muestras MAR y ZAR indica una reconstrucción con especies interlaminares de carbonatos y/o hidroxilos (Tichit et al. 1998, Sommer et al. 2013).

Los espectros infrarrojos de las hidrotalcitas MA, ZA y ZM (Fig. 2) muestran bandas en números de onda similares para los tres materiales y corresponden a valores reportados por otros autores (Sommer et al. 2013). Las bandas en 3400 y $1630 \mathrm{~cm}^{-1}$ corresponden a los grupos $\mathrm{OH}$ de las láminas y del agua presente en la región interlaminar de las hidrotalcitas (dos Reis et al. 2004). Las vibraciones en $1380 \mathrm{~cm}^{-1}$ se asocian con los aniones nitratos y carbonatos. La banda con máximo en $800 \mathrm{~cm}^{-1}$ se atribuye a la presencia de los cationes metálicos de $\mathrm{Mg}, \mathrm{Zn}$ y Al enlazados con oxígenos (Wei et al. 2005). En los materiales calcinados (MAC, ZAC y ZMAC) se aprecia una disminución de las bandas de los nitratos en $1380 \mathrm{~cm}^{-1}$ y las bandas de los $\mathrm{OH}$ en 3400 y $1630 \mathrm{~cm}^{-1}$, acorde con la descomposición de parte de estos compuestos con la temperatura de calcinación. Según lo previsto, las bandas correspondientes a los enlaces metal-oxígeno en $800 \mathrm{~cm}^{-1}$ se mantienen. Las muestras MAR, ZAR y ZMAR presentaron bandas similares a los materiales calcinados.

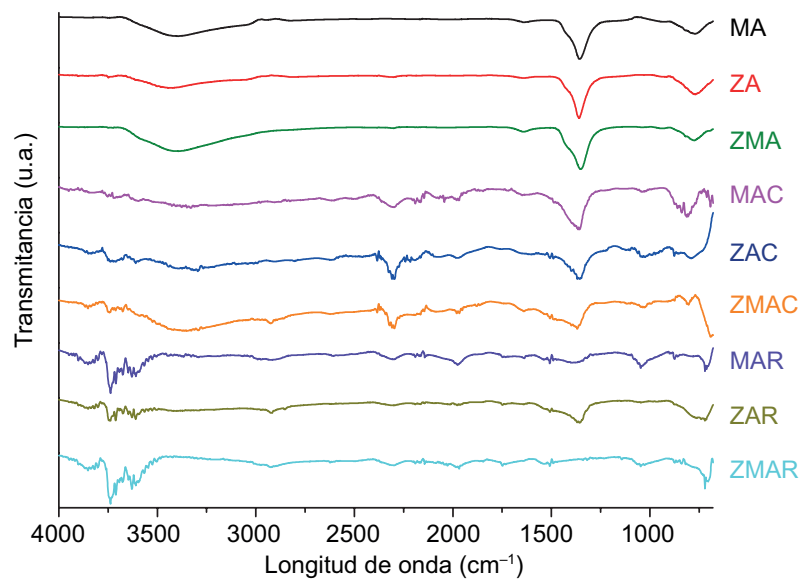

Fig. 2. Espectros infrarrojos de las hidrotalcitas (muestras MA, ZA y ZMA), de los óxidos mixtos derivados de las hidrotalcitas (MAC, ZAC y ZMAC) y de las hidrotalcitas reconstruidas (MAR, ZAR y ZMAR).
No obstante, se identifican las bandas atribuidas al grupo $\mathrm{OH}$ entre 3500 y $3750 \mathrm{~cm}^{-1}$ correspondientes al fenol (Tamura et al. 2006).

Las isotermas de adsorción-desorción de nitrógeno y las distribuciones de tamaños de poro de los materiales se muestran en la figura 3. Las isotermas de las hidrotalcitas MA, ZA y ZMA corresponden a isotermas tipo IV, según la clasificación de la International Union of Pure and Applied Chemistry (IUPAC), y con histéresis de tipo H3 características de materiales laminares como las arcillas (Prinetto et al. 2000). Asimismo, una pequeña diferencia aparece para la muestra MA en cuanto a la histéresis, que en este caso se presenta más ancha y con tramo final de desorción a presión relativa de cerca de 0.65 , característica de sólidos con gran cantidad de mesoporosos (Bergadà et al. 2007).

Con referencia a la distribución de tamaños de poros (Fig. 3b), la muestra MA presenta una curva con poros que van desde 50 hasta $250 \AA \AA$ de diámetro, mientras que los poros de las muestras ZA y ZMA se encuentran concentrados cerca de $30 \AA$ de diámetro. Las áreas superficiales específicas resultaron ser del orden de alrededor de $26 \mathrm{~m}^{2} / \mathrm{g}$ para las muestras dimetálicas MA y ZA, mientras que para la hidrotalcita trimetálica ZMA se obtuvo un valor de $4 \mathrm{~m}^{2} / \mathrm{g}$, debido probablemente al alto grado de compactación de las partículas en los aglomerados. En todos los casos, los valores concuerdan con los reportados por otros autores para hidrotalcitas nitratadas preparadas con métodos similares (Rivera et al. 2006, Sampieri et al. 2007, Sommer et al. 2010).

Respecto de los óxidos mixtos obtenidos por calcinación de las hidrotalcitas, éstos presentaron áreas específicas de 70,58 y $47 \mathrm{~m}^{2} / \mathrm{g}$ para las muestras MAC, ZAC y ZMAC, respectivamente. Estas áreas resultaron ser mucho mayores que las correspondientes a las hidrotalcitas, de acuerdo con valores reportados en la literatura (Zhang et al. 2010). Las isotermas de fisisorción de nitrógeno pertenecen al tipo IV con histéresis $\mathrm{H} 3$ en conformidad con estos tipos de materiales (Sommer et al. 2013); sin embargo, la distribución de tamaños de poros resultó ser más ancha para la muestra ZMAC, de 50 a $350 \AA$ de diámetro que para las otras dos, cuyos diámetros van de tres hasta 200 o $250 \AA$. Este comportamiento, inverso al de las hidrotalcitas, puede deberse a una mayor dispersión de los cationes en una red tridimensional de óxidos mixtos que su confinamiento en una red bidimensional de hidrotalcitas, siendo más fuerte el efecto a mayor cantidad de cationes de diferente naturaleza. 
(a)

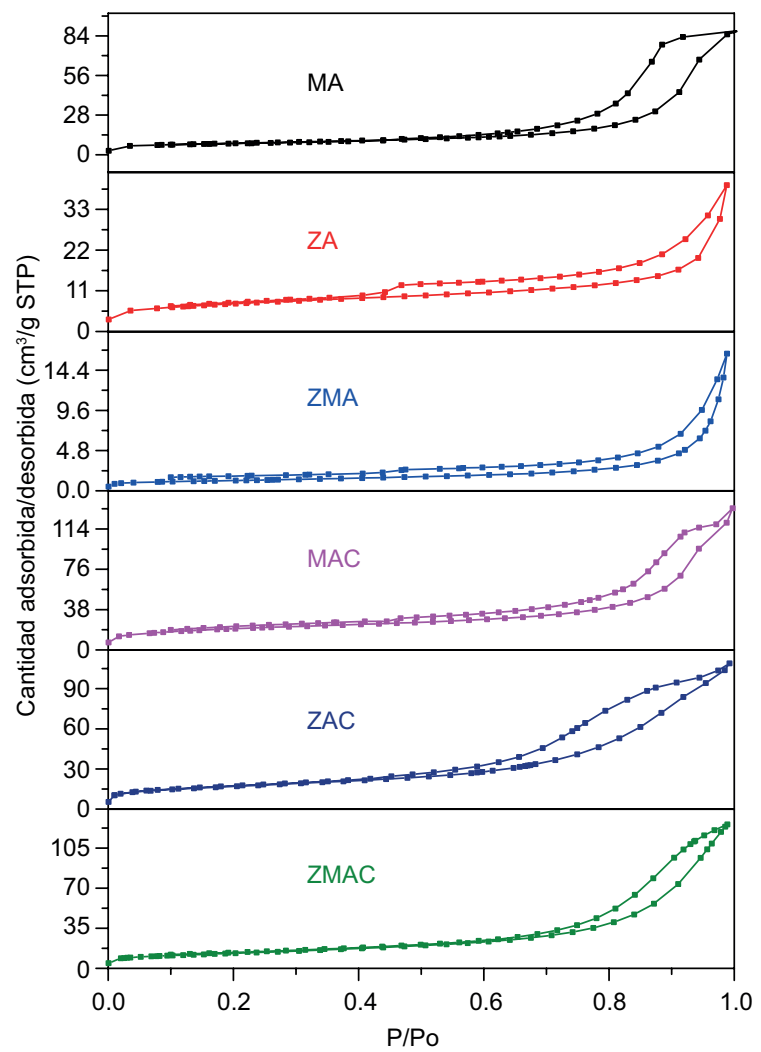

(b)

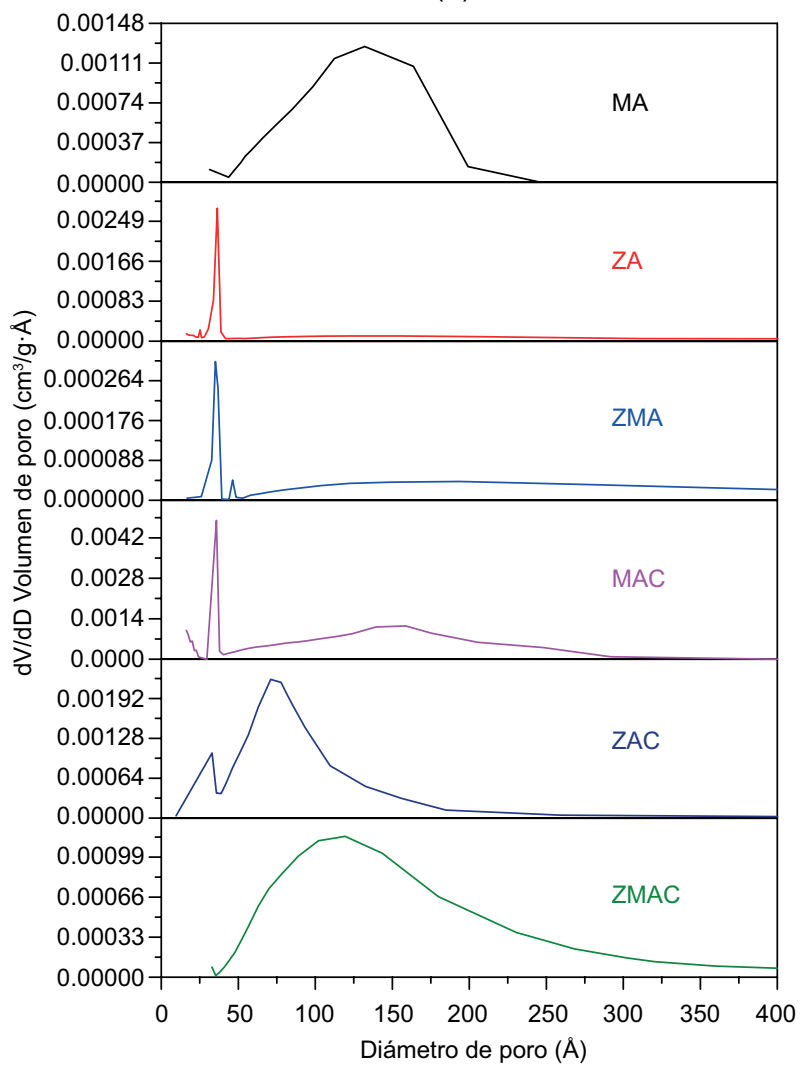

Fig. 3. (a) Isotermas de adsorción-desorción de $\mathrm{N}_{2}$ correspondientes a las muestras de hidrotalcitas y a los óxidos mixtos correspondientes. (b) Distribuciones de tamaños de poro de las hidrotalcitas y de los óxidos mixtos.

Para las muestras reconstruidas, las áreas específicas resultaron muy pequeñas, con valores menores a $2 \mathrm{~m}^{2} / \mathrm{g}$. Esto se debe probablemente a la obstrución de los poros por los compuestos orgánicos adsorbidos en los materiales (Lobo-Sánchez et al. 2018, Netzahualcoyotzi et al. 2016). Asimismo, las curvas de adsorción-desorción y de distribución de tamaños de poros no resultaron representativas, considerando que los resultados de las mediciones se encuentran en el orden de error de los análisis.

\section{Adsorción de BTEX-fenol}

En la figura 4 se muestran las cantidades adsorbidas de la mezcla BTEX-fenol por las hidrotalcitas, los óxidos mixtos y las hidrotalcitas reconstruidas. Para las hidrotalcitas MA y ZMA se observa que las capacidades de adsorción son similares y del orden de $1 \mathrm{a} 2 \mathrm{mg} / \mathrm{g}$, en tanto que la muestra ZA alcanza una adsorción de $5 \mathrm{mg} / \mathrm{g}$ a $1600 \mathrm{~min}$, al igual que las muestras calcinadas. Una gran diferencia en la cantidad adsorbida de los compuestos orgánicos ocurre con las hidrotalcitas reconstruidas, en las que

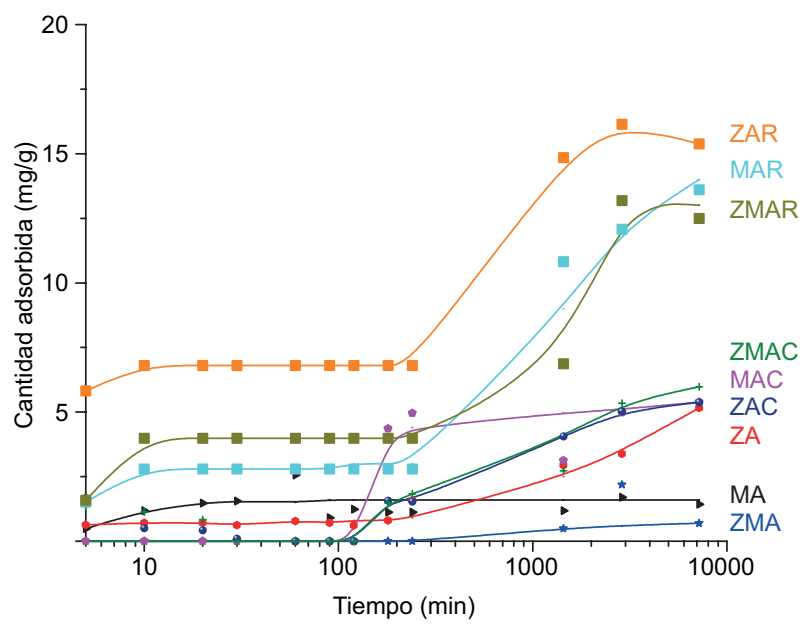

Fig. 4. Cantidad de BTEX-fenol en función del tiempo de contacto con las hidrotalcitas (muestras MA, ZA y ZMA), óxidos mixtos derivados de las hidrotalcitas (MAC, ZAC y ZMAC) e hidrotalcitas reconstruidas (MAR, ZAR y ZMAR). 
se reportan valores de 12 a $17 \mathrm{mg} / \mathrm{g}$ de material a los mismos 1600 min de tiempo de contacto. Además, se observa que existe un tiempo de inducción en el proceso de adsorción, que ocurre aproximadamente a 200 min prácticamente en todas las muestras.

Estas diferencias en cuanto a la capacidad y velocidad de adsorción deben atribuirse a las diferentes composiciones químicas de los materiales, que resultan en diferentes estructuras y modos de aglomeración de partículas y texturas, principalmente. Las velocidades y capacidades de retención de los BTEX-fenoles deben estar asociados con la difusión de éstos en los poros de los materiales y con las interacciones físicas y químicas con los sólidos. Para las hidrotalcitas, los factores determinantes fueron la composición química y el área específica, dado que la muestra que menos adsorbe (la ZMA) tiene ua área muy baja $\left(4 \mathrm{~m}^{2} / \mathrm{g}\right)$ en comparación con las otras dos $\left(26 \mathrm{~m}^{2} / \mathrm{g}\right)$; asimismo, aunque la muestra MA presenta una amplia distribución de tamaños de poro no es la que más adsorbe. La mayor retención ocurre para la hidrotalcita ZA y puede estar asociada a su carácter menos básico o más ácido (Ayala et al. 2011). A mayor carácter ácido, mayor positividad en los materiales y mayor será la interacción electrostática con los compuestos orgánicos con densidades de carga negativos originadas en los anillos aromáticos. Así, para estos materiales, ocurre una rápida saturación de contaminantes debido a la baja capacidad de retención de estos materiales.

Para los materiales calcinados MAC, ZAC y ZMAC las capacidades de adsorción resultaron similares, independientemente de las diferencias en su composición, estructura o textura. Estas similitudes pueden asociarse con las áreas superficiales similares de los materiales calcinados, que son del orden de $60 \mathrm{~m}^{2} / \mathrm{g}$. Asimismo, mejora la capacidad de adsorción de los óxidos mixtos, tomando en cuenta que adsorben dos o tres veces más que las hidrotalcitas precursoras; no obstante, si las comparamos con las hidrotalcitas reconstruidas, éstas resultaron mucho más eficientes. Se puede observar que los comportamientos de adsorción son muy diferentes a las otras muestras. Tienen una rápida adsorción de aproximadamente $5 \mathrm{mg} / \mathrm{g}$ al principio que posteriormente se estabiliza en cerca de 200 min para aumentar sus capacidades de adsorción a niveles de $14 \mathrm{mg} / \mathrm{g}$ a los $6000 \mathrm{~min}$. Además, una pequeña diferencia se observa entre estas tres muestras en cuanto a la capacidad de retención de los BTEX-fenoles, cuyo comportamiento sigue la composición química de las hidrotalcitas iniciales, donde la acidez atribuida a la presencia de zinc, en lugar del magnesio, favorece la retención de contaminantes, tanto a menores como a mayores tiempos de contacto.

Al comparar los comportamientos de adsorción de los BTEX-fenoles entre las diferentes muestras, queda claro que el proceso de adsorción por reconstrucción de las hidrotalcitas es el más eficiente, aunque podría ser más costoso. Cuando comparamos estos valores con los reportados por Jaynes y Vance (1999) en la sorción de BTEX con una arcilla tipo hectorita intercambiada con cationes orgánicos aromáticos (del orden de $15 \mathrm{mg} / \mathrm{g}$ para áreas superficiales de $60 \mathrm{~m}^{2} / \mathrm{g}$ ) se aprecia la eficiencia de los materiales reportados en este trabajo, además de que son materiales no tóxicos y amigables con el ambiente. Asimismo, considerando que estos materiales pueden regenerarse con facilidad, y teniendo en cuenta la alta capacidad de adsorción de las hidrotalcitas reconstruidas, sería muy factible la eliminación de contaminantes por calcinación al tiempo que se obtienen los óxidos mixtos, precursores de las hidrotalcitas reconstruidas. Por último, debido a que el proceso de calcinación para la obtención de óxidos mixtos no afecta a priori la reconstrucción de hidrotalcitas, ya que la composición química inorgánica se mantiene, éstas podrían utilizarse en varios ciclos de adsorción-reutilización.

\section{CONCLUSIONES}

Hidrotalcitas de diferentes composiciones y óxidos mixtos derivados de éstas fueron sintetizados para estudiar sus comportamientos frente a la adsorción de contaminantes aromáticos de tipo BTEXfenol, comúnmente presentes en aguas residuales. Las capacidades de adsorción se compararon con las de hidrotalcitas reconstruidas en presencias de los contaminantes BTEX-fenoles. Los resultados mostraron que los factores más importantes en el proceso de adsorción fueron la composición química de los materiales, la cual determinó la interacción sólido-adsorbato (determinada por el zinc) y de la estructura del material. La capacidad de adsorción de los óxidos mixtos fue de cerca de $5 \mathrm{mg} / \mathrm{g}$, cantidad ligeramente mayor que la retenida por las hidrotalcitas iniciales. No obstante, los materiales que mejor desempeñaron la función fueron las hidrotalcitas reconstruidas en presencia de los contaminantes, las cuales retuvieron cerca de $14 \mathrm{mg} / \mathrm{g}$. Considerando la alta capacidad de adsorción de estos materiales y la posibilidad de reconstruir sus estructuras, su uso en procesos de adsorción de contaminantes aromáticos resultaría ampliamente funcional, además de ser 
sólidos relativamente baratos, no tóxicos y amigables con el medio ambiente.

\section{REFERENCIAS}

Ayala A., Fetter G., Palomares E. y Bosch P. (2011). CuNi/ Al hydrotalcites synthesized in presence of microwave irradiation. Mater. Lett. 65 (11), 1663-1665. https://doi. org/10.1016/J.MATLET.2011.03.004

Bergadà O., Vicente I., Salagre P., Cesteros Y., Medina F. y Sueiras J.E. (2007). Microwave effect during aging on the porosity and basic properties of hydrotalcites. Microporous Mesoporous Mater. 101 (3), 363-373. https://doi.org/10.1016/j.micromeso.2006.11.033

Blanch-Raga N., Palomares A.E., Martínez-Triguero J., Puche M., Fetter G. y Bosch P. (2014). The oxidation of trichloroethylene over different mixed oxides derived from hydrotalcites. Appl. Catal. B Environ. 160-161 (1), 129-134. https://doi.org/10.1016/j.apcatb.2014.05.014

Chaillot D., Bennici S. y Brendlé J. (2020). Layered double hydroxides and LDH-derived materials in chosen environmental applications: A review. Environ. Sci. Pollut. Res. 28, 24375-24405. https://doi.org/10.1007/ s11356-020-08498-6

Dos Reis M.J., SilvérioF., Tronto J. y Valim J.B. (2004). Effects of $\mathrm{pH}$, temperature, and ionic strength on adsorption of sodium dodecylbenzenesulfonate into Mg-Al-CO3 layered double hydroxides. J. Phys. Chem. Solids 65 (2-3), 487-492. https://doi.org/10.1016/j. jpcs.2003.09.020

Dudek B., Kuśtrowski P., Białas A., Natkański P., Piwowarska Z., Chmielarz L., Kozak M. y Michalik M. (2012). Influence of textural and structural properties of $\mathrm{MgAl}$ and $\mathrm{MgZnAl}$ containing hydrotalcite derived oxides on $\mathrm{Cr}(\mathrm{VI})$ adsorption capacity. Mater. Chem. Phys. 132 (2-3), 929-936. https://doi.org/10.1016/J. MATCHEMPHYS.2011.12.037

Jaynes W.F. y Vance G.F. (1999). Sorption of benzene, toluene, ethylbenzene, and xylene (BTEX) compounds by hectorite clays exchanged with aromatic organic cations. Clays Clay Miner. 47 (3), 358-365. https:// doi.org/10.1346/CCMN.1999.0470312

Jurado-Dávila I., Rosset M., Perez-Lopez O. y AmaralFéris L. (2020). Removal of reactive red 120 in aqueous solution using Mg-hydrotalcites as adsorbents solids: Kinetics and isotherms. Rev. Int. Contam. Ambie. 36 (2), 443-453. https://doi.org/10.20937/rica.53539

León-Vallejo A.M., Velázquez-Herrera F.D., Sampieri Á., Landeta-Cortés G. y Fetter G. (2019). Study of layered double hydroxides as bactericidal materials against Corynebacterium ammoniagenes, a bacterium responsible for producing bad odors from human urine and skin infections. Appl. Clay Sci. 180, 105194. https://doi.org/10.1016/j.clay.2019.105194

Li X., Zhang L., Yang Z., Wang P., Yan Y. y Ran J. (2020). Adsorption materials for volatile organic compounds (VOCs) and the key factors for VOCs adsorption process: A review. Sep. Purif. Technol. 235, 116213. https://doi.org/10.1016/J.SEPPUR.2019.116213

Lobo-Sánchez M., Nájera-Meléndez G., Luna G., SeguraPérez V., Rivera J.A. y Fetter G. (2018). ZnAl layered double hydroxides impregnated with eucalyptus oil as efficient hybrid materials against multi-resistant bacteria. Appl. Clay Sci. 153, 61-69. https://doi. org/10.1016/j.clay.2017.11.017

Makhathini T. P. y Rathilal S. (2017). Investigation of BTEX compounds adsorption onto polystyrenic resin. South African J. Chem. Eng. 23, 71-80. https://doi. org/10.1016/J.SAJCE.2017.03.001

Mishra G., Dash B. y Pandey S. (2018). Layered double hydroxides: A brief review from fundamentals to application as evolving biomaterials. Appl. Clay Sci. 153, 172-186. https://doi.org/10.1016/j. clay.2017.12.021

Morales A., Hernández M., Pérez E., Guzmán A. y Lima E. (2018). Highly-polar layered double hydroxides are efficient adsorbents of low-concentrated trihalomethanes present in water. J. Mex. Chem. Soc. 62 (3), 99-109. https://doi.org/10.29356/jmcs.v62i3.557

Netzahualcoyotzi I., Galicia V., Rivera J.A., Fetter G. y Bosch P. (2016). Stabilization of hemoglobin in double layered hydroxides to be used in carbon monoxide bio-oxidation I-synthesis and characterization. Catal. Today 266, 212-218. https://doi.org/10.1016/j.cattod.2015.11.022

Pérez E., Ayele L., Getachew G., Fetter G., Bosch P., Mayoral A. y Díaz I. (2015). Removal of chromium(VI) using nano-hydrotalcite/SiO2 composite. J. Environ. Chem. Eng. 3 (3), 1555-1561. https://doi.org/10.1016/j. jece.2015.05.009

Prinetto F., Ghiotti G., Graffin P. y Tichit D. (2000). Synthesis and characterization of sol-gel $\mathrm{Mg} / \mathrm{Al}$ and $\mathrm{Ni}$ / Al layered double hydroxides and comparison with co-precipitated samples. Microporous Mesoporous Mater. 39 (1-2), 229-247. https://doi.org/10.1016/ S1387-1811(00)00197-9

Rivera J.A., Fetter G. y Bosch P. (2006). Microwave power effect on hydrotalcite synthesis. Microporous Mesoporous Mater. 89 (1-3), 306-314. https://doi. org/10.1016/j.micromeso.2005.10.041

Sampieri Á., Fetter G., Pfeiffer H. y Bosch P. (2007). Carbonate phobic (Zn,Mn)-Al hydrotalcite-like compounds. Solid State Sci., 9 (5), 394-403. https://doi. org/10.1016/j.solidstatesciences.2007.03.014 
Sampieri A., Pérez-Osorio G., Hernández-Espinosa M.Á., Ruiz-López I.I., Ruiz-Reyes M., Arriola-Morales J. y Narváez-Fernández R.I. (2018). Sorption of BTEX on a nanoporous composite of SBA-15 and a calcined hydrotalcite. Nano Converg. 5 (1), 21. https://doi. org/10.1186/s40580-018-0153-2

Sani T., Adem M., Fetter G., Bosch P. y Diaz I. (2016). Defluoridation performance comparison of nanohydrotalcite/hydroxyapatite composite with calcined hydrotalcite and hydroxyapatite. Water. Air. Soil Pollut. 227 (3), 1-8. https://doi.org/10.1007/s11270016-2786-2

Segura-Pérez V., Lobo-Sánchez M., Velázquez-Herrera F.D., Frías-Vázquez D.A., Reyes-Cervantes E. y Fetter G. (2020). Hydrotalcite/hydroxyapatite composites with high bacterial activity against clinical bacteria. A new alternative to prevent osteomyelitis diseases. Microporous Mesoporous Mater. 298, 110069. https:// doi.org/10.1016/j.micromeso.2020.110069

Smoláková L., Frolich K., Kocík J., Kikhtyanin O. y Čapek L. (2017). Surface properties of hydrotalcite-based $\mathrm{Zn}(\mathrm{Mg}) \mathrm{Al}$ oxides and their catalytic activity in aldol condensation of furfural with acetone. Ind. Eng. Chem. Res. 56 (16), 4638-4648. https://doi.org/10.1021/acs. iecr.6b04927

Sommer A., Rivera J.A., Fetter G. y Bosch P. (2006). Utilización de arcillas aniónicas sintetizadas por irradiación de microondas en la condensación aldólica de la acetona. Rev. Mex. Ing. Quím. 5 (3), 245-251.

Sommer A., Fetter G., Bosch P. y Lara V.H. (2010). New template effect in hydrotalcite synthesis. Nodular vs layered morphologies. Clays Clay Miner. 58 (3), 340350. https://doi.org/10.1346/CCMN.2010.0580305

Sommer A., Romero A., Fetter G., Palomares E. y Bosch P. (2013). Exploring and tuning the anchorage of chlorophyllin molecules on anionic clays. Catal. Today 212, 186-193. https://doi.org/10.1016/j.cattod.2013.03.014

Tamura H., Chiba J., Ito M., Takeda T., Kikkawa S., Mawatari Y. y Tabata M. (2006). Formation of hydrotalcite in aqueous solutions and intercalation of ATP by anion exchange. J. Colloid Interface Sci. 300 (2), 648-654. https://doi.org/10.1016/j.jcis.2006.04.007
Tichit D., Naciri-Bennani M., Figueras F., Tessier R. y Kervennal J. (1998). Aldol condensation of acetone over layered double hydroxides of the meixnerite type. Appl. Clay Sci. 13 (5-6), 401-415. https://doi. org/10.1016/S0169-1317(98)00035-0

Velázquez-Herrera F.D., Fetter G., Rosato V., Pereyra A.M. y Basaldella E.I. (2018). Effect of structure, morphology and chemical composition of $\mathrm{Zn}-\mathrm{Al}, \mathrm{Mg}$ / $\mathrm{Zn}-\mathrm{Al}$ and $\mathrm{Cu} / \mathrm{Zn}-\mathrm{Al}$ hydrotalcites on their antifungal activity against $A$. niger. J. Environ. Chem. Eng. 6 (2), 3376-3383. https://doi.org/10.1016/j.jece.2018.04.069

Velázquez-Herrera F.D. y Fetter G. (2020). Hydrotalcites with heterogeneous anion distributions: a first approach to produce new materials to be used as vehicles for a successive delivery of compounds. Clay Miner. 55 (1), 31-39. https://doi.org/10.1180/clm.2020.2

Velázquez-Herrera F.D., González-Rodal D., Fetter G. y Pérez-Mayoral E. (2020). Enhanced catalytic performance of highly mesoporous hydrotalcite/SBA-15 composites involved in chromene multicomponent synthesis. Microporous Mesoporous Mater. 309. https://doi.org/10.1016/j.micromeso.2020.110569

Wei M., Wang J., He J., Evans D.G. y Duan X. (2005). In situ FT-IR, in situ HT-XRD and TPDE study of thermal decomposition of sulfated $\beta$-cyclodextrin intercalated in layered double hydroxides. Microporous Mesoporous Mater. 78 (1), 53-61. https://doi.org/10.1016/j. micromeso.2004.09.016

Zarazúa-Aguilar Y., Paredes-Carrera S.P., ValenzuelaZapata M.A. y Sánchez-Ochoa J.C. (2018). Cr (VI) and naftalene simultaneous degradation using layered double hydroxides CuZnGa. Rev. Mex. Ing. Quím. 17 (2), 679-691. https://doi.org/10.24275/uam/izt/dcbi/ revmexingquim/2018v17n2/zarazua

Zhang Z., Zhang Y., Wang Z. y Gao X. (2010). Catalytic performance and mechanism of potassium-promoted $\mathrm{Mg}-\mathrm{Al}$ hydrotalcite mixed oxides for soot combustion with $\mathrm{O}_{2}$. J. Catal. 271 (1), 12-21. https://doi. org/10.1016/j.jcat.2010.01.022 